\title{
Finite groups of units of finite characteristic rings
}

\author{
Ilaria Del Corso ${ }^{1}$ • Roberto Dvornicich ${ }^{1}$
}

Received: 21 April 2017 / Accepted: 4 September 2017 / Published online: 13 September 2017

(C) Fondazione Annali di Matematica Pura ed Applicata and Springer-Verlag GmbH Germany 2017

\begin{abstract}
Fuchs (Abelian groups, Pergamon, Oxford, 1960, Problem 72) asked the following question: which groups can be the group of units of a commutative ring? In the following years, some partial answers have been given to this question in particular cases. The aim of the present paper is to address Fuchs' question when $A$ is a finite characteristic ring. The result is a pretty good description of the groups which can occur as group of units in this case, equipped with examples showing that there are obstacles to a "short" complete classification. As a by-product, we are able to classify all possible cardinalities of the group of units of a finite characteristic ring, so to answer Ditor's question (Ditor in Am Math Mon 78(5):522-523, 1971).
\end{abstract}

Keywords Commutative algebra · Group theory · Fuchs' problem · Units groups · Finite characteristic rings

Mathematics Subject Classification 13M99 - 16U60

\section{Introduction}

In [7, Problem 72] Fuchs posed the following problem:

Characterize the groups which are the groups of all units in a commutative and associative ring with identity.

In the following years, this question and related ones have been considered by many authors. A first result is due to Gilmer [8], who classified the finite commutative rings $A$ with the property that the group of units $A^{*}$ is cyclic, classifying as a by-product also the possible cyclic groups that arise in this case.

Ilaria Del Corso

ilaria.delcorso@unipi.it

1 Universita degli Studi di Pisa, Pisa, Italy 
Further steps in the study of Fuchs' Problem came from the results by Hallet and Hirsch [9], and subsequently by Hirsch and Zassenhauss [10], combined with [2]. From their results, one can deduce necessary conditions for a finite group to be the group of units of a reduced and torsion free ring.

Later on, Pearson and Schneider [11] combined the result of Gilmer and the result of Hallet and Hirsch to describe explicitly all possible finite cyclic groups that can occur as $A^{*}$ for a commutative ring $A$.

In 1971 Ditor [4], in view of the difficulty of dealing with the general Fuchs Problem, posed the following less general question:

Which whole numbers can be the number of units of a ring?

In his paper, he answered this question in the easier case of odd integers.

Other instances on this subject were considered also by other authors, like Eldridge and Fischer [6], who considered rings with the descending chain condition, or Dolkan [5], who classified the finite rings $A$ for which $A^{*}$ is a finite group whose order is either a prime power or not divisible by 4 . Recently, Chebolu and Lockridge [1] have studied Fuchs' problem in a different setting and have been able to classify the indecomposable abelian groups that are realizable as the group of units of a ring.

The aim of the present paper is to address Fuchs' and Ditor's questions when $A$ is a finite characteristic ring. We call an abelian group realizable if it is the group of units of some finite characteristic ring.

We first show that the general problem can be reduced to the study of the group of units of finite local rings. In Theorem 3.1 and Corollary 3.2, we give necessary conditions for an abelian group to be realizable. The conditions found in Theorem 3.1 allow to produce infinite families of non-realizable groups (see Proposition 4.1). As a direct consequence, we reobtain the classification of cyclic units groups already found by Gilmer in [8] (see Corollary 4.2).

On the other hand, in the paper, we also give positive answers; in fact, we exhibit large classes of groups that are realizable. For example, in Propositions 4.3 we show that, for $p>2$, all groups of the form $\mathbb{F}_{p^{\lambda}}^{*} \times P^{\lambda}$ are realizable, for all $\lambda \geq 1$ and for all abelian $p$-group $P$. Proposition 4.3 contains a similar result for $p=2$.

Moreover, in Sect. 5, we give further examples which are not included in Propositions 4.3 and 4.8. Both the propositions and the construction of the examples need the case of rings with characteristic a power of 2 to be treated separately. This is mainly due to the particular behavior of $\left(\mathbb{Z} / p^{n} \mathbb{Z}\right)^{*}$ for $p=2$.

Our results allow to exhibit infinite families of "new" examples of realizable groups (see Remark 4.6) and infinite families of non-realizable groups (see Proposition 4.1), reducing considerably the space between necessary and sufficient conditions for a group to be realizable. In view of the examples in Sect. 5, we believe that a more precise statement of necessary and sufficient conditions would be very complicated.

Moreover, we are able to completely answer Ditor's question for finite characteristic rings (see Corollary 3.3) and to construct, for each possible cardinality $n$, a finite characteristic ring $A$ with $\left|A^{*}\right|=n$.

As a final remark, we point out that our results include all previously known facts for the case under consideration; some results already proved in [8] and in [5] have been derived as particular cases in our paper.

In a forthcoming paper [3], we investigate Fuchs' and Ditor's Problems in the case of characteristic 0 rings and we obtain a complete classification of the groups of units of reduced rings. 


\section{Notation and preliminary results}

Let $A$ be ring with 1 of finite characteristic. As usually, $A^{*}$ will denote its multiplicative group, which we shall assume finite and abelian throughout the paper.

We recall that a ring is called reduced if its nilradical is trivial, namely, if it does not contain non-zero nilpotents.

Remark 2.1 Let $n=\operatorname{char}(A)$, then $\mathbb{Z} / n \mathbb{Z}$ is the fundamental subring of $A$. Let $B=$ $\mathbb{Z} / n \mathbb{Z}\left[A^{*}\right]$; then $B \subseteq A$, and $B^{*}=A^{*}$.

We also note that the rings $A$ and $B$ have the same nilradical; in fact, the nilradical of $B$ is obviously contained in the nilradical of $A$; on the other hand, if $a \in A$ is such that $a^{n}=0$ for some $n \in \mathbb{N}$, then $1+a \in A^{*} \subseteq B^{*}$, so $a \in B$. In particular, $A$ is reduced if and only if $\mathbb{Z} / n \mathbb{Z}\left[A^{*}\right]$ is reduced.

If $A$ is a domain, then its characteristic is a prime number $p$ and $\mathbb{Z} / p \mathbb{Z}\left[A^{*}\right]$ is a finite field.

In this paper, we are interested in determining the possible abelian groups that occur as groups of units of a finite characteristic ring: as a consequence of the previous remark, we may restrict our study to commutative and finite rings.

In the following, we shall make repeated use of the following proposition:

Proposition 2.2 Let A be a commutative ring, let $\mathfrak{N}$ denote its nilradical and let $\mathfrak{I}$ be an ideal of A contained in $\mathfrak{N}$. Then the sequence

$$
1 \rightarrow 1+\mathfrak{I} \hookrightarrow A^{*} \stackrel{\phi}{\rightarrow}(A / \mathfrak{I})^{*} \rightarrow 1
$$

where $\phi(x)=x+\mathfrak{I}$, is exact.

Proof Clearly

$$
1 \rightarrow 1+\mathfrak{I} \rightarrow A^{*} \rightarrow A^{*} /(1+\mathfrak{I}) \rightarrow 1
$$

is exact. Moreover, the map $\Phi: A^{*} \rightarrow(A / \mathfrak{I})^{*}$ defined by $x \mapsto x+\mathfrak{I}$ is a homomorphism with kernel equal to $1+\mathfrak{I}$. Further, if $x+\mathfrak{I} \in(A / \mathfrak{I})^{*}$ and $(x+\mathfrak{I})(y+\mathfrak{I})=1+\mathfrak{I}$ then $x y \in 1+\mathfrak{I} \subseteq A^{*}$, so $x \in A^{*}$ and $\Phi$ is surjective, so $A^{*} /(1+\mathfrak{I}) \cong(A / \mathfrak{I})^{*}$.

The case when $\mathfrak{I}=\mathfrak{N}$ is particularly interesting since, as we shall see in the following, for finite characteristic rings the exact sequence (1) always splits in this case (see Theorem 3.1). However, there are cases when the exact sequence does not split.

\section{General results}

As noted in the previous section, we may suppose that $A$ is finite and commutative, so we will assume it in the following. Under this assumption, we have that $A$ is artinian, then by the structure theorem of artinian rings, $A$ is isomorphic to a direct product of artinian local rings,

$$
A \cong A_{1} \times \cdots \times A_{s},
$$

and hence

$$
A^{*} \cong A_{1}^{*} \times \cdots \times A_{s}^{*}
$$


This reduces the problem to the study of the cardinality and the structure of the group of units of a finite local ring. We recall that the characteristic of such a ring is a prime power. The following theorem describes the groups of units in this case.

Theorem 3.1 Let $p$ be a prime, and let $(A, \mathfrak{m})$ be a finite local ring of characteristic a power of $p$, such that $A^{*}$ is finite. Then

$$
A^{*} \cong \mathbb{F}_{p^{\lambda}}^{*} \times H
$$

where $A / \mathfrak{m} \cong \mathbb{F}_{p^{\lambda}}$ and $H=1+\mathfrak{m}$ is an abelian p-group. Moreover, the filtration $\left\{1+\mathfrak{m}^{i}\right\}_{i \geq 1}$

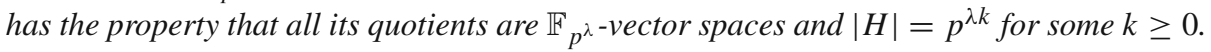

Conversely, for each prime $p$, for each $\lambda \geq 1$ and for each $k \geq 0$ there exists a local artinian ring with characteristic a power of $p$ such that $A^{*} \cong \mathbb{F}_{p^{\lambda}}^{*} \times H$ with $|H|=p^{\lambda k}$.

Finally, when restricting to reduced local artinian rings, the groups of units that can be obtained are precisely the multiplicative groups of finite fields.

Proof In our hypothesis, we have that the nilradical $\mathfrak{N}$ coincides with $\mathfrak{m}$, so the exact sequence in (1) can be written as

$$
1 \rightarrow 1+\mathfrak{m} \rightarrow A^{*} \rightarrow(A / \mathfrak{m})^{*} \rightarrow 1 .
$$

Consider the finite chain

$$
\mathfrak{m} \supset \mathfrak{m}^{2} \supset \cdots \supset \mathfrak{m}^{r}=\{0\} .
$$

We have that, for each $i=1, \ldots r-1$, the quotient $\mathfrak{m}^{i} / \mathfrak{m}^{i+1}$ is a $A / \mathfrak{m}$-vector space, and hence has cardinality $p^{\lambda k_{i}}$ for some positive integer $k_{i}$. Putting $k=\sum_{i=1}^{r-1} k_{i}$, we obtain that $|\mathfrak{m}|=p^{\lambda k}$ and hence $|1+\mathfrak{m}|=p^{\lambda k}$.

We can now apply Schur-Zassenhaus Theorem (see $[12,9.12])$ to the exact sequence in (3): it follows that $A^{*} \cong \mathbb{F}_{p^{\lambda}}^{*} \times(1+\mathfrak{m})$ and equation (2) follows with $H=1+\mathfrak{m}$.

Moreover, the isomorphism $\mathfrak{m}^{i} / \mathfrak{m}^{i+1} \rightarrow\left(1+\mathfrak{m}^{i}\right) /\left(1+\mathfrak{m}^{i+1}\right)$ induced by the map $x \mapsto 1+x$ ensures that the quotients of the filtrations $\left\{\mathfrak{m}^{i}\right\}_{i \geq 1}$ and $\left\{1+\mathfrak{m}^{i}\right\}_{i \geq 1}$ are isomorphic, hence $\left(1+\mathfrak{m}^{i}\right) /\left(1+\mathfrak{m}^{i+1}\right)$ is a $\mathbb{F}_{p^{\lambda}}$-vector space for each $i$.

Conversely, let $\lambda \geq 1, k \geq 0$ and let $f(t) \in\left(\mathbb{Z} / p^{k+1} \mathbb{Z}\right)[t]$ be a monic polynomial of degree $\lambda$ such that its reduction modulo $p, \bar{f}(t)$, is irreducible. Define

$$
R=\frac{\left(\mathbb{Z} / p^{k+1} \mathbb{Z}\right)[t]}{(f(t))} ;
$$

clearly $R$ is a local artinian ring and its maximal ideal $\mathfrak{m}$ is generated by the class of $p$. As above, $R^{*} \cong \mathbb{F}_{p^{\lambda}}^{*} \times(1+\mathfrak{m})$ and it is easy to check that $|1+\mathfrak{m}|=|\mathfrak{m}|=p^{\lambda k}$.

We conclude by noticing that the statement about reduced rings is clear, since a reduced local artinian ring is a field.

We summarize the results for a general ring of finite characteristic in the following corollary, which also retrieves some known results as particular cases.

Corollary 3.2 Let $A$ be a ring of finite characteristic. Then $A^{*}$ is a finite the product of groups as in (2), where each prime $p \mid \operatorname{char}(A)$, gives at least one factor.

Moreover, the finite abelian groups that occur as group of units of reduced rings of finite characteristic are exactly the finite products of multiplicative groups of finite fields.

Finally, if $A$ is a domain, then $A^{*}$ is the multiplicative group of a finite field.

Proof As observed at the beginning of this section, if $A$ is a ring of finite characteristic with a finite abelian group of units, then $A^{*} \cong A_{1}^{*} \times \cdots \times A_{s}^{*}$, where $A_{i}$ are local artinian finite rings. Then, the information on $A^{*}$ can be derived by applying Theorem 3.1 to each $A_{i}$. 
Theorem 3.1 allows to completely answer the question raised by Ditor [4] in the case of finite characteristic rings. In fact, we have the following

Corollary 3.3 The possible values for $\left|A^{*}\right|$ when char $(A)$ is finite are all products of numbers of type $\left(p^{\lambda}-1\right) p^{\lambda k}$, where $p \mid \operatorname{char}(A), \lambda \geq 1$ and $k \geq 0$. In this product there exists at least a factor of type $\left(p^{\lambda}-1\right) p^{\lambda k}$ for each prime divisor of $\operatorname{char}(A)$.

We note that the examples in the proof of Theorem 3.1 allow the explicit construction of a ring $A$ with $\left|A^{*}\right|=n$, for each possible $n$.

For the sake of completeness, we include the following corollary, which is not new and can be found for example in [4].

Corollary 3.4 The finite abelian groups of odd order that occur as groups of units of a ring $A$ are the finite products of groups of type $\mathbb{F}_{2^{\lambda}}^{*}$. In particular, if $\left|A^{*}\right|=p$ is prime, then $p$ is a Mersenne prime.

Proof It is enough to note that if $\left|A^{*}\right|$ is odd, then necessarily $\operatorname{char}(A)>0$ and to apply Corollary 3.3.

\section{Families of realizable and non-realizable groups}

Theorem 3.1 does not solve completely the question of classifying the abelian $p$-groups $H$ which occur as $1+\mathfrak{m}$ in a local artinian ring. However, in view of the constraints on the filtration of the group $H$, our results show that the group $H$ cannot be any $p$-group if $\lambda>1$, nor any group of order a power of $p^{\lambda}$; in fact, we have the following

Proposition 4.1 Let $p$ be a prime, let $k$ and $\lambda$ be positive integers and let $H$ be an abelian p-group of cardinality $p^{\lambda k}$. If there exists a local artinian ring $A$ such that $A^{*} \cong \mathbb{F}_{p^{\lambda}}^{*} \times H$, then the exponent of $H$ is at most $p^{k}$.

Proof By Theorem 3.1, a necessary condition in order that $H$ can appear in (2) is that $H$

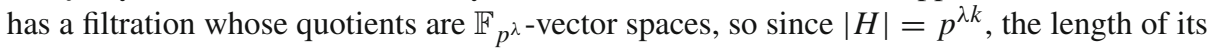
filtration can be at most $k$, and every element of $H$ must have order at most $p^{k}$.

Example 1 The group $H \cong \mathbb{Z} / p^{3} \mathbb{Z} \times(\mathbb{Z} / p \mathbb{Z})^{2 \lambda-3}$ does not occur as $1+\mathfrak{m}$ if $A / \mathfrak{m}=\mathbb{F}_{p^{\lambda}}$, since $|H|=p^{2 \lambda}$ and $H$ contains elements of order $p^{3}$.

From the previous proposition, it is easy to obtain a complete classification of finite cyclic groups of units which arise in the case of finite characteristic rings. This result is not new (as we have already noted, it was firstly proved by Gilmer [8]); however, we include it since, at least for the case $p>2$, it is an immediate consequence of Proposition 4.1. Actually, the proof for the case $p=2$ requires some more effort, which, however, well highlights the importance of the condition on the filtration in Theorem 3.1.

Corollary 4.2 A finite cyclic group is the group of units of a local artinian ring of characteristic a power of a prime $p$ if and only if its order is:
a. $p^{\lambda}-1$ for $\lambda \geq 1$;
b. $(p-1) p^{k}$ for $k \geq 1$ and $p>2$;
c. 2;
d. 4 . 
A finite cyclic group is the group of units of a finite characteristic ring if and only if its order is the product of a set of pairwise coprime integers of the previous list.

Proof By Theorem 3.1, using the same notation, we have $A^{*} \cong \mathbb{F}_{p^{\lambda}}^{*} \times H$ and in particular $H$ must be cyclic: Proposition 4.1 implies $|H|=1$ or $\lambda=1$. It follows that $\left|A^{*}\right|$ can only be either $p^{\lambda}-1$ with $\lambda \geq 1$ or $(p-1) p^{k}$ for $k \geq 1$. For $p>2$ all these groups occur, for example with $A=\mathbb{F} p^{\lambda}$ and $A=\mathbb{Z} / p^{k+1} \mathbb{Z}$.

For $p=2$ we obtain all groups of order $2^{\lambda}-1$ by choosing $A=\mathbb{F}_{2^{\lambda}}$. We obtain the groups of order 2 and 4 by choosing $A=\mathbb{Z} / 4 \mathbb{Z}$ or $(\mathbb{Z} / 2 \mathbb{Z})[x] /\left(x^{3}\right)$.

On the other hand, when the characteristic of $A$ is a power of 2 , no other case is possible. In fact, since $(\mathbb{Z} / 8 \mathbb{Z})^{*}$ is not cyclic, necessarily the characteristic of $A$ can only be 2 or 4 . We also note that in both cases $2 x=0$ for all $x \in \mathfrak{m}$ (if not, $\pm 1 \pm x$ would be 4 distinct elements with the same square, and this is not possible in a cyclic group). Assume that $\left|A^{*}\right|=2^{k}$; then $\lambda=1$ and $H=1+\mathfrak{m}$ is cyclic of order $2^{k}$. Let $1+\mu$ be a generator of $1+\mathfrak{m}$. The only filtration of $1+\mathfrak{m}$ which satisfies the condition of Theorem 3.1 is

$$
\left\{(1+\mathfrak{m})^{2^{i}}\right\}_{1 \leq i \leq k} \text {. }
$$

It follows that the length of the filtration in (4) is $r-1=k$ and $\mu^{r}=0$. Now, since $2 \mu=0$, and $1+\mu$ has order $2^{k}$, we have $(1+\mu)^{2^{k-1}}=1+\mu^{2^{k-1}} \neq 1$. Therefore, $2^{k-1}<r=k+1$, so $k=1$ or $k=2$.

Finally, the statement about a general ring $A$ of finite characteristic follows recalling that its group of units $A^{*}$ is the product of groups of units $A_{i}^{*}(i=1, \ldots, r)$ of local artinian rings, so it is cyclic if and only if all the $A_{i}^{*}$ 's are cyclic and their orders are pairwise coprime.

On the positive side, we are able to exhibit a large class of new groups that are realizable, in the sense that they cannot be in general obtained as a product of realizable cyclic groups (see Remark 4.6). In Proposition 4.3, we show that for each $p>2, \lambda \geq 1$ and for each abelian $p$-group $P$ one can construct a ring $A$ for which $H=P^{\lambda}$. In particular, for $\lambda=1$ all abelian $p$-groups are possible.

The case $p=2$ is exceptional and needs to be treated separately: a class of groups (most of them new) which can be realized in this case is given in Proposition 4.8.

In the next section, we will exhibit examples of realizable groups which are not covered by Propositions 4.3 and 4.8 .

Proposition 4.3 Let $p>2$ be a prime. For each $\lambda \geq 1$ and for each finite abelian $p$-group $P$ there exists a local artinian ring $A$ such that

$$
A^{*} \cong \mathbb{F}_{p^{\lambda}}^{*} \times P^{\lambda}
$$

Proof Consider the decomposition of the group $P$ as a product of cyclic groups $P \cong$ $\mathbb{Z} / p^{a_{0}} \mathbb{Z} \times \mathbb{Z} / p^{a_{1}} \mathbb{Z} \times \cdots \times \mathbb{Z} / p^{a_{r}} \mathbb{Z}$ with $a_{0} \geq a_{1} \geq \cdots \geq a_{r}$, and let $f(t) \in\left(\mathbb{Z} / p^{a_{0}+1} \mathbb{Z}\right)[t]$ be a monic polynomial of degree $\lambda$ such that its reduction modulo $p, \bar{f}(t)$, is irreducible. As in the proof of Theorem 3.1, define

$$
R=\frac{\left(\mathbb{Z} / p^{a_{0}+1} \mathbb{Z}\right)[t]}{(f(t))}
$$

and

$$
A=\frac{R\left[x_{1}, \ldots, x_{r}\right]}{\left(p^{a_{i}} x_{i}, x_{i} x_{j}\right)_{1 \leq i, j \leq r}} .
$$


We already observed that $R$ is a local artinian ring with maximal ideal $\mathfrak{m}_{R}=(p)$. We note that also $A$ is a local artinian ring with maximal ideal $\mathfrak{m}_{A}=\left(p, \bar{x}_{1}, \ldots, \bar{x}_{r}\right)$; in fact,

$$
A / \mathfrak{m}_{A} \cong R / \mathfrak{m}_{R} \cong \mathbb{F}_{p^{\lambda}}
$$

and $\mathfrak{m}_{A}$ is the unique prime ideal of $A$ since all its elements are nilpotent.

We will show that $A^{*} \cong \mathbb{F}_{p^{\lambda}}^{*} \times P^{\lambda}$.

Let $\mathfrak{I}=\left(\bar{x}_{1}, \ldots, \bar{x}_{r}\right) \subset \mathfrak{m}_{A}$. By Proposition 2.2 we have the exact sequence

$$
1 \rightarrow 1+\mathfrak{I} \hookrightarrow A^{*} \stackrel{\phi}{\rightarrow}(A / \mathfrak{I})^{*} \rightarrow 1
$$

Now, it is clear that $(A / \mathfrak{I})^{*} \cong R^{*} \subset A^{*}$ so the exact sequence splits and $A^{*} \cong R^{*} \times(1+\mathfrak{I})$.

We note that $1+\mathfrak{I} \cong \mathfrak{I}$ since the map $x \mapsto 1+x$ is a group isomorphism. Now, $\mathfrak{I} \cong\left(\mathbb{Z} / p^{a_{1}} \mathbb{Z} \times \cdots \times \mathbb{Z} / p^{a_{r}} \mathbb{Z}\right)^{\lambda}$ since, as an abelian group, it is generated by the elements $\left\{\bar{t}^{j} \bar{x}_{i}\right\}_{0 \leq j<\lambda, 1 \leq i \leq r}$ and $\bar{t}^{j} \bar{x}_{i}$ has additive order $p_{i}^{a}$, since $a_{1} \leq a_{0}+1$. On the other hand, by Theorem 3.1, we know that $R^{*} \cong \mathbb{F}_{p^{\lambda}} \times\left(1+\mathfrak{m}_{R}\right)$, so we are left to prove that $1+$ $\mathfrak{m}_{R} \cong\left(\mathbb{Z} / p^{a_{0}} \mathbb{Z}\right)^{\lambda}$. We note that $R$ is a free $\left(\mathbb{Z} / p^{a_{0}+1} \mathbb{Z}\right)$-module with basis $1, \bar{t}, \ldots, \bar{t}^{\lambda-1}$, so $R \cong\left(\mathbb{Z} / p^{a_{0}+1} \mathbb{Z}\right)^{\lambda}$ as a group, hence $\mathfrak{m}_{R}=p R \cong\left(\mathbb{Z} / p^{a_{0}} \mathbb{Z}\right)^{\lambda}$. Thus, we are reduced to show that $1+\mathfrak{m}_{R} \cong \mathfrak{m}_{R}$. Since both are $p$-groups, it is enough to prove that the subgroup $\left(1+\mathfrak{m}_{R}\right)_{p^{l}}$ of elements of exponent $p^{l}$ of $1+\mathfrak{m}_{R}$ has the same cardinality of the subgroup $\left(\mathfrak{m}_{R}\right)_{p^{l}}$ of elements of exponent $p^{l}$ of $\mathfrak{m}_{R}$. This is a direct consequence of the following lemma.

Lemma 4.4 Let $\mu \in \mathfrak{m}_{R}=p R$ and let $l \geq 0$. Then,

$$
(1+\mu)^{p^{l}}=1 \Longleftrightarrow p^{l} \mu=0 .
$$

Proof By the binomial theorem,

$$
(1+\mu)^{p^{l}}=1+p^{l} \mu+\sum_{k=2}^{p^{l}}\left(\begin{array}{l}
p^{l} \\
k
\end{array}\right) \mu^{k} .
$$

We claim that, if $\mu \in p^{i} R(i \geq 1)$ and hence $p^{l} \mu \in p^{l+i} R$, then $\left(\begin{array}{c}p^{l} \\ k\end{array}\right) \mu^{k} \in p^{l+i+1} R$, for all $k \geq 2$. In fact, clearly $\mu^{k} \in p^{i k} R$; moreover, it is a classical result that if $p^{c} \| k$, then $p^{l-c} \|\left(\begin{array}{c}p^{l} \\ k\end{array}\right)$, so, since $p>2$ and $k \geq 2$, we have that $p^{l-k+2} \operatorname{divides}\left(\begin{array}{c}p^{l} \\ k\end{array}\right)$ and

$$
p^{l+(i-1) k+2} \mid\left(\begin{array}{c}
p^{l} \\
k
\end{array}\right) \mu^{k} .
$$

Now, $l+(i-1) k+2 \geq l+i+1$ since this is equivalent to $(i-1)(k-1) \geq 0$ (recall that $i \geq 1$ and $k \geq 2$ ), and the claim follows.

In conclusion, if $p^{l} \mu=0$ (namely, if $l+i \geq a_{0}+1$ ), then $\left(\begin{array}{c}p^{l} \\ k\end{array}\right) \mu^{k}=0$ also for all $k \geq 2$, so $(1+\mu)^{p^{l}}=1$; on the other hand, if $p^{l} \mu \neq 0$, then there exists $i$ with $l+i \leq a_{0}$ such that $p^{l} \mu \in p^{l+i} R \backslash p^{l+i+1} R$, so

$$
(1+\mu)^{p^{l}} \equiv 1+p^{l} \mu \not \equiv 1 \quad\left(\bmod p^{l+i+1}\right) .
$$

The case $\lambda=1$ of Proposition 4.3 immediately gives the following 
Corollary 4.5 Let $p>2$ be a prime. For each finite abelian $p$-group $P$ there exists a finite local ring A such that

$$
A^{*} \cong \mathbb{F}_{p}^{*} \times P .
$$

Remark 4.6 Proposition 4.3 produces infinite examples of "new" groups of units. In fact, if $p>2$ is not a Mersenne prime, and $P$ is a non cyclic abelian $p$-group, then the group $\mathbb{F}_{p^{\lambda}}^{*} \times P^{\lambda}$ is realizable but is not a product of cyclic realizable groups. In fact, it is enough to observe that $2^{a}-1=p^{b}$ has no solution in view of the famous Catalan-Mihhilescu Theorem.

Remark 4.7 Proposition 4.3 cannot be generalized to $p=2$. In fact, putting $p=2$ and $\lambda=1$ we would obtain that all abelian 2-groups are groups of units but, as recalled in Corollary 4.2 , this is not true already for cyclic groups.

The peculiarity of the case $p=2$ lies in the fact that the group $\left(\mathbb{Z} / 2^{a} \mathbb{Z}\right)^{*}$ is not cyclic in general. However, in the following proposition, we exhibit a large class of groups of units of rings with characteristic a power of 2 .

Proposition 4.8 Let $P$ be a finite abelian 2-group and let $2^{a}$ be its exponent. Then, for each integer $a_{0} \geq a-1$ and for each $\lambda \geq 1$ there exists a local artinian ring $A$ such that

$$
A^{*} \cong \mathbb{F}_{2^{\lambda}}^{*} \times \mathbb{Z} / 2 \mathbb{Z} \times \mathbb{Z} / 2^{a_{0}-1} \mathbb{Z} \times\left(\mathbb{Z} / 2^{a_{0}} \mathbb{Z}\right)^{\lambda-1} \times P^{\lambda}
$$

Proof Consider the decomposition of $P$ as a product of cyclic groups, $P \cong \mathbb{Z} / 2^{a_{1}} \mathbb{Z} \times \cdots \times$ $\mathbb{Z} / 2^{a_{r}} \mathbb{Z}$ with $a_{1} \geq \cdots \geq a_{r}$, so $a=a_{1}$. Let $f(t) \in \mathbb{Z}[t]$ be a monic polynomial of degree $\lambda$ such that its reduction modulo $2, \bar{f}(t)$, is irreducible. As in Proposition 4.3, define

$$
R=\frac{\left(\mathbb{Z} / 2^{a_{0}+1} \mathbb{Z}\right)[t]}{(f(t))}
$$

and

$$
A=\frac{R\left[x_{1}, \ldots x_{r}\right]}{\left(2^{a_{i}} x_{i}, x_{i} x_{j}\right)_{1 \leq i, j \leq r}} ;
$$

$R$ and $A$ are local artinian rings with maximal ideals $\mathfrak{m}_{R}=2 R$ and $\mathfrak{m}_{A}=\left(2, \bar{x}_{1}, \ldots, \bar{x}_{r}\right)$, respectively. We will show that $A$ has the required group of units. Again, letting $\mathfrak{I}=$ $\left(\bar{x}_{1}, \ldots, \bar{x}_{r}\right) \subset \mathfrak{m}_{A}$, we can argue as in the proof of Proposition 4.3 and get $A^{*} \cong R^{*} \times(1+\mathfrak{I})$ and $1+\mathfrak{I} \cong \mathfrak{I} \cong P^{\lambda}$.

Moreover, $R^{*} \cong \mathbb{F}_{2^{\lambda}}^{*} \times(1+2 R)$. Then we have to study the group $1+2 R$ : as in Proposition 4.3 we have $R \cong\left(\mathbb{Z} / 2^{a_{0}+1} \mathbb{Z}\right)^{\lambda}$ as a group, so $2 R \cong\left(\mathbb{Z} / 2^{a_{0}} \mathbb{Z}\right)^{\lambda}$, but, contrary to the case $p>2,1+2 R$ is not always isomorphic to $2 R$. $2^{\lambda}$.

When $a_{0}=1$, it is easy to see that the group $1+2 R$ is elementary abelian of cardinality

Assume now $a_{0} \geq 2$. To determine the structure of $1+2 R$ it is enough to know the structure of its subgroup $(1+2 R)^{2}$ and of the quotient $(1+2 R) /(1+2 R)^{2}$, since this quotient has the same number of generators as the group $1+2 R$.

We begin by studying the quotient. Clearly, it is an elementary abelian 2-group, so it is enough to determine its order; now, $\left|(1+2 R) /(1+2 R)^{2}\right|=|\operatorname{ker} \varphi|$ where $\varphi:(1+2 R) \rightarrow$ $(1+2 R)^{2}$ is defined by $u \mapsto u^{2}$. Let $1+2 \overline{p(t)} \in 1+2 R$, where $\overline{p(t)} \in R$ and $\operatorname{deg} p(t)<\lambda$. We have

$$
(1+2 \overline{p(t)})^{2}=1 \Longleftrightarrow 4 \overline{p(t)}(1+\overline{p(t)})=0 \Longleftrightarrow \overline{p(t)}(1+\overline{p(t)}) \in 2^{a_{0}-1} R .
$$


Since $R$ is a local ring, one between $\overline{p(t)}$ and $1+\overline{p(t)}$ is invertible, so either $\overline{p(t)} \in 2^{a_{0}-1} R$ or $1+\overline{p(t)} \in 2^{a_{0}-1} R$ and actually exactly one of these two relations holds. Hence, $\operatorname{ker} \varphi=$ $2^{a_{0}} R \cup 1+2^{a_{0}} R$ and

$$
\left|(1+2 R) /(1+2 R)^{2}\right|=|\operatorname{ker} \varphi|=2\left|2^{a_{0}} R\right|=2^{\lambda+1} .
$$

Next, to study $(1+2 R)^{2}$, we find it convenient to study first the larger group $1+4 R$. We observe that $|(1+2 R) /(1+4 R)|=2^{\lambda}$ (recall that $\left.a_{0} \geq 2\right)$, hence $\left|(1+4 R) /(1+2 R)^{2}\right|=2$. As in Proposition 4.3 , we prove that $1+4 R$ is isomorphic to $4 R \cong\left(\mathbb{Z} / 2^{a_{0}-1} \mathbb{Z}\right)^{\lambda}$ by showing that the two groups have the same number of elements of exponent $2^{l}$ for all $l$. This is a consequence of the following slight variation of Lemma 4.4.

Lemma 4.9 Let $\mu \in 4 R$ and let $l \geq 0$. Then,

$$
(1+\mu)^{2^{l}}=1 \Longleftrightarrow 2^{l} \mu=0 .
$$

Proof Let $\mu \in 2^{i} R$ with $i \geq 2$. Consider the expansion of $(1+\mu)^{2^{l}}$. Clearly $2^{l+i} \| 2^{l} \mu$; as in the proof of Lemma 4.4 it is enough to show that $2^{l+i+1}$ divides $\left(\begin{array}{c}2^{l} \\ k\end{array}\right) \mu^{k}$ for $k \geq 2$. In this case, $2^{l-k+1}$ divides $\left(\begin{array}{c}2^{l} \\ k\end{array}\right)$, so

$$
2^{l-k+1+i k} \mid\left(\begin{array}{l}
2^{l} \\
k
\end{array}\right) \mu^{k}
$$

Now, $l-k+1+i k \geq l+i+1$ since this is equivalent to $i(k-1) \geq k$ and $i, k \geq 2$.

Putting everything together we get

$$
(1+2 R)^{2} \cong \mathbb{Z} / 2^{a_{0}-2} \mathbb{Z} \times\left(\mathbb{Z} / 2^{a_{0}-1} \mathbb{Z}\right)^{\lambda-1}
$$

and

$$
(1+2 R) \cong \mathbb{Z} / 2 \mathbb{Z} \times \mathbb{Z} / 2^{a_{0}-1} \mathbb{Z} \times\left(\mathbb{Z} / 2^{a_{0}} \mathbb{Z}\right)^{\lambda-1}
$$

\section{Further examples}

In this section, we give examples showing that the groups $H$ obtained in Propositions 4.3 and 4.8 do not exhaust all realizable groups.

Example 2 Let $p>2, f(t)$ be a monic polynomial of degree 2 which is irreducible modulo $p$ and let

$$
A=\frac{\left(\mathbb{Z} / p^{2} \mathbb{Z}\right)[t, x]}{\left(f(t), p x^{2}, x^{p-1}+p\right)} .
$$

We claim that $A$ is a finite local artinian ring and, denoting by $\mathfrak{m}$ its maximal ideal, we have $A^{*} \cong \mathbb{F}_{p^{2}} \times(1+\mathfrak{m})$ and $1+\mathfrak{m}$ is not the square of a group, so this group of units does not belong to the set of groups described in Proposition 4.3. Defining

$$
R=\frac{\left(\mathbb{Z} / p^{2} \mathbb{Z}\right)[t]}{(f(t))}, \quad B=\frac{R[x]}{\left(x^{p-1}+p\right)}
$$


we have

$$
A=\frac{B}{\left(p \bar{x}^{2}\right)} .
$$

Denoting by $\bar{x}$ the class of $x$ in $B$, we have

$$
B=\oplus_{i=0}^{p-2} R \bar{x}^{i}
$$

as an $R$-module. It follows that, as an $R$-module, the ideal $\left(p \bar{x}^{2}\right)$ is equal to $\oplus_{i=2}^{p-2} R p \bar{x}^{i}$, hence

$$
A=\frac{\oplus_{i=0}^{p-2} R \bar{x}^{i}}{\oplus_{i=2}^{p-2} R p \bar{x}^{i}} \cong R^{2} \oplus\left(\frac{R}{p R}\right)^{p-3}
$$

as an $R$-module, and $|A|=p^{2 p+2}$. Clearly $A$ is a finite local artinian ring, since $B$ is such; its maximal ideal is $\mathfrak{m}=(p, \overline{\bar{x}})=(\overline{\bar{x}})$, where $\overline{\bar{x}}$ denotes the class of $\bar{x}$ in $A$. This gives the $R$-module isomorphism

$$
\mathfrak{m}=p R \oplus R \overline{\bar{x}} \oplus_{i=2}^{p-2} R \overline{\bar{x}}^{i} \cong R \oplus\left(\frac{R}{p R}\right)^{p-2},
$$

so, $|\mathfrak{m}|=p^{2 p}$ and $A / \mathfrak{m} \cong \mathbb{F}_{p^{2}}$, i.e., in the notation of Proposition $4.3, \lambda=2$.

We show that

$$
1+\mathfrak{m} \cong \mathbb{Z} / p^{2} \mathbb{Z} \times(\mathbb{Z} / p \mathbb{Z})^{2 p-2}
$$

and hence it is not the square of a group. In fact, it is enough to prove that in $1+\mathfrak{m}$ there are exactly $p^{2 p-1}$ elements of exponent $p$.

Let $a \in 1+\mathfrak{m}$, then $a$ can be written as $a=1+a_{0} p+a_{1} \overline{\bar{x}}+\cdots+a_{p-2} \overline{\bar{x}}^{p-2}$ where $a_{1} \in R$ is uniquely determined, and $a_{i} \in R$ is uniquely determined modulo $p$ if $i \neq 1$. Then $a^{p}=1+p\left(a_{1}-a_{1}^{p}\right) \overline{\bar{x}}=1$ if and only if the reduction of $a_{1}$ modulo $p$ belongs to $\mathbb{F}_{p}$. The elements $a$ verifying this condition are $p^{2 p-1}$ since there are $p^{2}$ possibilities for $a_{i}$ if $i \neq 1$, and $p^{3}$ possibilities for $a_{1}$, and the claim follows.

Example 3 For $p=2$ and $a_{0} \geq 3$, consider the ring

$$
A=\frac{\left(\mathbb{Z} / 2^{a_{0}+1} \mathbb{Z}\right)[t, x]}{\left(t^{2}+t+1,4 x, x^{2}+2 x\right)} .
$$

We show that $A$ is a finite local artinian ring, but $A^{*}$ does not belong to the set of groups described in Proposition 4.8. Theorem 3.1 guarantees that $A^{*}=(A / \mathfrak{m})^{*} \times(1+\mathfrak{m})$, where $\mathfrak{m}$ is the maximal ideal of $A$.

Similarly to above, we can consider the ring

$$
R=\left(\mathbb{Z} / 2^{a_{0}+1} \mathbb{Z}\right)[t] /\left(t^{2}+t+1\right) ;
$$

we have $\mathfrak{m}_{R}=\mathfrak{m} \cap R=2 R$ and $A / \mathfrak{m}=R \mathfrak{m}_{R}=\mathbb{F}_{4}$, so in the previous notation $\lambda=2$. As seen in the proof of Proposition 4.8, we have that

$$
1+\mathfrak{m}_{R}=(1+\mathfrak{m}) \cap R^{*} \cong \mathbb{Z} / 2 \mathbb{Z} \times \mathbb{Z} / 2^{a_{0}-1} \mathbb{Z} \times \mathbb{Z} / 2^{a_{0}} \mathbb{Z}
$$

is a subgroup of $1+\mathfrak{m}$. 
Denote by $\bar{x}$ the class of $x$ in $A$; arguing as in Example 2, we have

$$
\mathfrak{m}=(2, \bar{x})=2 R \oplus \bar{x} R \cong R / 2^{a_{0}} R \oplus R / 4 R
$$

as an $R$-module, and $|1+\mathfrak{m}|=|\mathfrak{m}|=2^{2 a_{0}+4}$.

To determine the decomposition of $1+\mathfrak{m}$ as a product of cyclic groups, we begin by counting its elements of exponent 2. Let $\mu \in \mathfrak{m}$, then $\mu=2 \rho+\sigma \bar{x}$, where $\rho, \sigma \in R$ and are uniquely determined modulo $2^{a_{0}} R$ and modulo $4 R$, respectively. Taking into account that $4 \bar{x}=0$, we have

$$
\begin{aligned}
(1+\mu)^{2}=1 & \Longleftrightarrow 4\left(\rho+\rho^{2}\right)+2\left(\sigma+\sigma^{2}\right) \bar{x}=0 \\
& \Longleftrightarrow \rho+\rho^{2} \in 2^{a_{0}-1} R \text { and } \sigma+\sigma^{2} \in 2 R .
\end{aligned}
$$

Now, $\rho+\rho^{2} \in 2^{a_{0}-1} R$ if and only if $\rho \in 2^{a_{0}-1} R \cup\left(-1+2^{a_{0}-1} R\right)$ and each of the two classes has 4 representatives in $R / 2^{a_{0}} R$, so there are 8 possibilities for $\rho$. The condition $\sigma+\sigma^{2} \in 2 R$ can be rewritten as $\pi(\sigma)+\pi(\sigma)^{2}=0$, where $\pi: R / 4 R \rightarrow R / 2 R \cong \mathbb{F}_{4}$ is the canonical projection. This means that $\pi(\sigma) \in \mathbb{F}_{2}$, so there are 8 possibilities for $\sigma$. In conclusion, there are $2^{6}$ elements of exponent 2 in $1+\mathfrak{m}$. This yields that the decomposition of $1+\mathfrak{m}$ has 6 cyclic factors. Taking into account that $1+\mathfrak{m}$ has order $2^{2 a_{0}+4}, 6$ cyclic factors and a subgroup isomorphic to $\mathbb{Z} / 2 \mathbb{Z} \times \mathbb{Z} / 2^{a_{0}-1} \mathbb{Z} \times \mathbb{Z} / 2^{a_{0}} \mathbb{Z}$, it is immediate to see that

$$
1+\mathfrak{m} \cong(\mathbb{Z} / 2 \mathbb{Z})^{3} \times \mathbb{Z} / 4 \mathbb{Z} \times \mathbb{Z} / 2^{a_{0}-1} \mathbb{Z} \times \mathbb{Z} / 2^{a_{0}} \mathbb{Z}
$$

so, for $a_{0} \geq 3$, the group $A^{*}$ does not belong to the set of groups described in Proposition 4.8 .

\section{References}

1. Chebolu, S.K., Lockridge, K.: Fuchs' problem for indecomposable abelian groups. J. Algebra 438, 325336 (2015)

2. Corner, A.L.S.: Every countable reduced torsion-free ring is an endomorphism ring. Proc. Lond. Math. Soc. 13(3), 687-710 (1963)

3. Del Corso, I., Dvornicich, R.: On Fuchs' Problem about the group of units of a ring (2017, submitted)

4. Ditor, S.Z.: On the group of units of a ring. Am. Math. Mon. 78(5), 522-523 (1971)

5. Dolžan, D.: Groups of units in a finite ring. J. Pure Appl. Algebra 170(2-3), 174-183 (2002)

6. Eldridge, K.E., Fisher, I.: Dcc rings with a cyclic group of units. Duke Math. J. 34, 243-248 (1967)

7. Fuchs, L.: Abelian Groups, 3rd edn. Pergamon, Oxford (1960)

8. Gilmer, R.W.: Finite rings with a cyclic group of units. Am. J. Math. 85, 447-452 (1963)

9. Hallet, J.T., Hirsch, K.A.: Torsion-free groups having finite automorphism groups. J. Algebra 2, 287-298 (1965)

10. Hirsch, K.A., Zassenhaus, H.: Finite automorphism groups of torsion free groups. J. Lond. Math. Soc. 41, 545-549 (1966)

11. Pearson, K.R., Schneider, J.E.: Rings with a cyclic group of units. J. Algebra 16, 243-251 (1970)

12. Robinson, D.J.S.: A Course in the Theory of Groups. Graduate Texts in Mathematics, vol. 80, 2nd edn. Springer, New York (1995) 